Check for updates

Cite this: RSC Adv., 2019, 9, 3847

Received 21st December 2018 Accepted 14th January 2019

DOI: 10.1039/c8ra10471a

rsc.li/rsc-advances

\section{Effects of Ga substitution on electronic and thermoelectric properties of gapless semiconductor $\mathrm{V}_{3} \mathrm{Al}$}

\author{
Xiaorui Chen, Yuhong Huang, Jing Liu, Hongkuan Yuan (D) and Hong Chen (D)*
}

\section{Introduction}

High efficiency thermoelectric (TE) materials are significant components for power generation devices that can convert waste heat into electricity. The conversion efficiency of TE materials is generally characterized by the dimensionless figure of merit defined as $z T=S^{2} \sigma T / \kappa,{ }^{\mathbf{1} 2}$ where $S, \sigma, T$, and $\kappa$ are the Seebeck coefficient, the electrical conductivity, the absolute temperature, and the total thermal conductivity respectively. The total thermal conductivity $(\kappa)$ is composed of two parts, $\kappa=$ $\kappa_{\mathrm{e}}+\kappa_{\mathrm{l}}$, where $\kappa_{\mathrm{e}}\left(\kappa_{\mathrm{l}}\right)$ is the carrier (lattice) thermal conductivity. The thermally-driven electrical performance of TE materials is measured by the power factor $\left(\mathrm{PF}=S^{2} \sigma\right)$. To improve $z T$, one has to enhance PF and/or weaken $\kappa$. The main challenge is how to make an improvement in the value of $z T$ owing to the interdependency of the transport parameters $\left(S, \sigma\right.$, and $\left.\kappa_{\mathrm{e}}\right)$. The electronic thermal conductivity $\left(\kappa_{\mathrm{e}}\right)$ is positively related to the electrical conductivity $(\sigma)$ by the Wiedemann-Franz law and a counteracted relationship between $S$ and $\sigma$ is often observed. Therefore, the optimization of $z T$ is a great and challenging task for obtaining highly effective TE materials.

Among promising thermoelectric materials, half-Heusler alloys have been mostly studied due to their large power factor, mechanical properties and thermal stability. ${ }^{3}$ The semiconducting half-Heusler alloys have shown to exhibit large Seebeck coefficients $\left(\sim 300 \mu \mathrm{V} \mathrm{K} \mathrm{K}^{-1}\right)$ at room temperature and moderate electrical conductivities $\left(\sim 1000-10000 \Omega^{-1} \mathrm{~cm}^{-1}\right),{ }^{4-7}$ which are attributed to their narrow bands associated to high effective masses. Nevertheless, their relatively high thermal

School of Physical Science and Technology, Southwest University, Chongqing 400715, China.E-mail: chenh@swu.edu.cn conductivities lead to their low thermoelectric efficiencies. In order to decrease the lattice thermal conductivity which is the predominant contribution to the thermal conductivity, ${ }^{8}$ some efforts have been made by synthesizing compounds with complicated structures by nanostructuring, forming nanocomposites, and isoelectronic alloying, ${ }^{3}$ which enhances the phonon scattering by point defects due to mass differences (mass fluctuations) and size differences (strain field impurities) between the impurity atoms and the host atoms. Typically, a $z T$ $\sim 1$ can be achieved for the nanostructured p-type $\mathrm{Nb}_{0.6} \mathrm{Ti}_{0.4^{-}}$ $\mathrm{FeNiSb}_{0.95} \mathrm{Sn}_{0.05}$ composition near $973 \mathrm{~K}$ with a cost-effective mass-production nanocomposite approach. ${ }^{9}$ The thermal conductivity $(\kappa)$ of a $\mathrm{TiNiSn}_{0.95} \mathrm{Sb}_{0.05}$ sample synthesized by ball milling and shock compaction can drop from 10.6 to $3.7 \mathrm{~W} \mathrm{~m}^{-1}$ $\mathrm{K}^{-1}$ by reducing the grain size diameter of the sample from $\sim 10$ to $1 \mu \mathrm{m} .{ }^{10}$ The isoelectronic substitution of $\mathrm{Zr}$ for Hf in HfNiSnbased alloys remarkably reduced the lattice thermal conductivity and a $z T \sim 1.0$ was achieved at $1000 \mathrm{~K}$ for $\mathrm{Hf}_{0.6} \mathrm{Zr}_{0.4^{-}}$ $\mathrm{NiSn}_{0.98} \mathrm{Sb}_{0.02}$ (ref. 11) and an even higher $z T \sim 1.2$ for $\mathrm{Hf}_{0.6} \mathrm{Zr}_{0.4} \mathrm{NiSn}_{0.995} \mathrm{Sb}_{0.005}{ }^{12}$ Record-high $z T$ values have been obtained for n-type $\mathrm{Ti}_{0.5} \mathrm{Zr}_{0.25} \mathrm{Hf}_{0.025} \mathrm{NiSn}_{0.998} \mathrm{Sb}_{0.002}(z T \sim 1.5$ at $700 \mathrm{~K},{ }^{13} z T \sim 1.2$ at $830 \mathrm{~K}$ (ref. 14)) and for p-type $\mathrm{FeNb}_{0.86}{ }^{-}$ $\mathrm{Hf}_{0.14} \mathrm{Sb}\left(z T \sim 1.5\right.$ at $1200 \mathrm{~K}$ (ref. 15)) and $\mathrm{FeTi}_{0.2} \mathrm{Nb}_{0.8} \mathrm{Sb}(z T \sim$ 1.1 at $1100 \mathrm{~K}$ (ref. 16)). In addition, semiconducting fullHeuslers with 24 valence electrons are other remarkable candidates for moderate temperature thermoelectric modules. Although only low $z T$ values of $0.1-0.2$ were achieved ${ }^{17-19}$ in the full-Heusler alloys due to lower density of states close to the Fermi level compared to half-Heusler alloys, a significant edge of improvement exists if the thermal conductivity is reduced and $\mathrm{PF}$ is further increased via manipulation of the electronic properties. Hole-type doping by Mn substitution at the Fe site 
and electron-type doping by $\mathrm{Sb}$ substitution at the $\mathrm{Sn}$ site have been explored in $\mathrm{Fe}_{2}$ TiSn samples prepared by arc melting plus spark plasma sintering or annealing. The remarkable $\mathrm{PF} \sim$ $0.4 \mathrm{~mW} \mathrm{~m}^{-1} \mathrm{~K}^{-2}$ in $\mathrm{Fe}_{2-x} \mathrm{Mn}_{x} \mathrm{TiSn}(x=0.0185)^{20}$ and $\mathrm{PF} \sim 1.3$ $\mathrm{mW} \mathrm{m}{ }^{-1} \mathrm{~K}^{-2}$ in $\mathrm{FeTiSn}_{1-x} \mathrm{Sb}_{x}(x=0.1)^{21}$ at room temperature indicated a more than double increase in $\mathrm{PF}$ at room temperature in the doped samples compared to that of the undoped one, which has been attributed to a strong resonant level induced by doped atoms near the Fermi level. Hole and isoelectronic doped $\mathrm{Fe}_{2} \mathrm{VAl}$ by Ta substitution both at the $\mathrm{Al}^{19,22,23}$ and at the $\mathrm{V}$ site ${ }^{23,24}$ have been demonstrated to exhibit power factors in the range of 5-9 $\mathrm{mW} \mathrm{m}^{-1} \mathrm{~K}^{-2}$ above room temperature.

The ternary Heusler phases often display defects including vacancy, antisite and swap defects, specifically, there is a natural tendency toward off-stoichiometry, predictable from thermodynamic theory, which may affect and even destroy the semiconductivity and transport properties, ${ }^{25}$ as observed experimentally in half-Heusler $\mathrm{ZrNiSn}^{26}$ and full-Heusler $\mathrm{Fe}_{2}$ TiSn. ${ }^{21,27}$ In contrast to the ternary Heuslers, the binary Heusler alloys have slight disorder compared to the ternary ones because there is only one type of transition metal. In particular, $\mathrm{V}_{3} \mathrm{Al}$ has been recently predicted theoretically ${ }^{28}$ and then confirmed experimentally ${ }^{29}$ to be an antiferromagnetic (AF) gapless semiconductor (GS) with a stable cubic $\mathrm{D0}_{3}$ structure. The thermoelectric behavior of $\mathrm{D}_{3}-\mathrm{V}_{3} \mathrm{Al}$ has been studied and

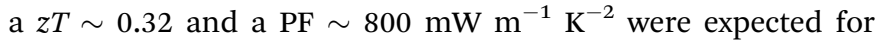
p-type $\mathrm{AF}-\mathrm{DO}_{3}-\mathrm{V}_{3} \mathrm{Al}$ at a temperature of $500 \mathrm{~K} \cdot{ }^{30}$ Although the thermoelectric performance and efficiency of $\mathrm{V}_{3} \mathrm{Al}$ are largely enhanced compared to the full-Heusler alloys, it cannot compete with the efficiency of conventional power generators or refrigerators mainly due to its high thermal conductivities. To further explore this enhanced performance, we have made some attempts to reduce the thermal conductivity by substituting $\mathrm{Al}$ with the isoelectronic element, $\mathrm{Ga}$, in a $\mathrm{V}_{3} \mathrm{Al}$ alloy. For this purpose, the electronic and thermoelectric properties of $\mathrm{V}_{3}$ $\mathrm{Al}_{1-x} \mathrm{Ga}_{x}(x=0.25,0.5,0.75$ and 1$)$ have been systematically studied. In addition, the mechanical stability and robustness of the doped $\mathrm{V}_{3} \mathrm{Al}$ compounds were asserted by calculating elastic constants $\left(C_{i j}\right)$ and moduli (bulk modulus $B$, shear modulus $G$, and Young's modulus $E$ ). The thermoelectric properties of $\mathrm{V}_{3^{-}}$$\mathrm{Al}_{1-x} \mathrm{Ga}_{x}(x=0.25,0.5,0.75$ and 1$)$ were predicted by applying the semi-classical Boltzmann transport theory.

\section{Computational method and process}

The $\mathrm{D}_{3}$-type $\mathrm{Ga}$ doped $\mathrm{V}_{3} \mathrm{Al}$-based compounds $\mathrm{V}_{3} \mathrm{Al}_{1-x} \mathrm{Ga}_{x}$ have face-centered cubic structures and have the space group of $F m \overline{3} m .^{28,29,31,32}$ In the Wyckoff coordination, $\mathrm{Al}$ atoms are located at the $4 \mathrm{a}(0,0,0)$ site, whereas the position of the $\mathrm{V}$ atoms is complicated. There are $12 \mathrm{~V}$ atoms in the unit cell of the $\mathrm{V}_{3} \mathrm{Al}$ compound, 8 of which occupy the $4 \mathrm{~b}(0.5,0.5,0.5)$ and the other $4 \mathrm{~V}$ atoms are at $4 \mathrm{c}(0.25,0.25,0.25)$ and $4 \mathrm{~d}(0.75,0.75,0.75)$ (labeled $\mathrm{V}_{1}$ and $\mathrm{V}_{2}$ ). The doping concentrations of $x=0.25,0.5$, 0.75 and 1 in the $\mathrm{V}_{3} \mathrm{Al}_{1-x} \mathrm{Ga}_{x}$ compounds mean that $1,2,3$ or 4 $\mathrm{Al}$ atoms are substituted by $\mathrm{Ga}$ atoms in the $\mathrm{V}_{3} \mathrm{Al}$ unit cell. All properties of $\mathrm{V}_{3} \mathrm{Al}_{1-x} \mathrm{Ga}_{x}(x=0.25,0.5,0.75$ and 1$)$ were calculated from first-principles within the framework of the projector augmented wave (PAW) method $^{33}$ as conducted using the package VASP. ${ }^{34-36}$ The generalized gradient approximation (GGA) and the Perdew-Burke-Ernzerhof (PBE) ${ }^{37}$ were adopted for the exchange-correlation energy of electrons. For all the structures, the plane wave cut-off energy and a Monkhorst-Pack $k$-mesh sample in the whole Brillouin zone were $560 \mathrm{eV}$ and 15 $\times 15 \times 15$ respectively, which are the same as those used in our previous work for $\mathrm{V}_{3} \mathrm{Al} .{ }^{30}$ For $\mathrm{V}, \mathrm{Al}$, and $\mathrm{Ga}, 3 \mathrm{~d}^{3} 4 \mathrm{~s}^{2}, 3 \mathrm{~s}^{2} 3 \mathrm{p}^{1}$, and $4 \mathrm{~s}^{2} 4 \mathrm{p}^{1}$ states are considered as the valence states. The atom position is fully relaxed until the magnitude of the energy change and the force acting on each atom are smaller than $10^{-5} \mathrm{eV}$ and $0.01 \mathrm{eV} \AA^{-1}$. As shown in the previous work, ${ }^{30} \mathrm{~V}_{3} \mathrm{Al}$ is more stable in the $\mathrm{AF}$ phase, and therefore all the calculations were conducted for $\mathrm{AF} \mathrm{DO}_{3}$ Ga-doped $\mathrm{V}_{3} \mathrm{Al}$.

To examine the mechanical stability of $\mathrm{V}_{3} \mathrm{Al}_{1-x} \mathrm{Ga}_{x}$, we need to evaluate the elastic constants, which can be obtained by the stress-strain method. For a cubic crystal, there are only three independent components, namely, $C_{11}, C_{12}$, and $C_{44}$. Among them, $C_{11}$ represents the elasticity in length, while $C_{12}$ and $C_{44}$ characterize the elasticity in shape. These elastic constants can be determined by computing the stress generated by applying a small strain to an optimized unit cell. More details of the calculations can be found in the literature. ${ }^{38-40}$ The bulk modulus $(B)$, shear modulus $(G)$, and Young's modulus $(E)$ can be expressed according to the elastic constants by the VoigtReuss-Hill (VRH) approximation. ${ }^{41}$ Thus, the mechanical stability of $\mathrm{V}_{3} \mathrm{Al}_{1-x} \mathrm{Ga}_{x}$ can be judged by the following conditions: ${ }^{\mathbf{4 2 , 4 3}}$

$$
C_{11}+2 C_{12}>0, C_{44}>0, C_{11}-C_{12}>0 \text {. }
$$

Electronic transport coefficients are predicted by using the semi-classical Boltzmann theory within the accurate band structures. In this approach, the electrical conductivity $(\sigma)$ and electronic thermal conductivity $\left(\kappa_{\mathrm{e}}\right)$ are dependent on the relaxation time $(\tau)$. To calculate thermoelectric properties, we have to first calculate the relaxation time which is usually very complicated since it depends on phonon scattering, carrier scattering, and defect scattering mechanisms. Unlike some of the previous theoretical works where $\sigma$ and $\kappa_{\mathrm{e}}$ were calculated based on a constant relaxation time ${ }^{\mathbf{4 4 , 4 5}}$ or an experimental nonconstant relaxation time, ${ }^{\mathbf{4 6 , 4 7}}$ we determine relaxation time by using the combination of deformation potential (DP) theory and effective mass approximation which produces a reliable evaluation of electronic transport coefficients for FeNbSb-based half-Heusler thermoelectric materials compared with experimental data. ${ }^{48}$ As the lattice constant is much smaller in comparison with the wavelength of thermally activated carriers, the electron-acoustic phonon coupling is the dominant mechanism. ${ }^{49}$ The scheme is fortunately simplified. Accordingly, the relaxation time for a three-dimensional system can be calculated by the following formula: ${ }^{50}$

$$
\tau=\frac{2 \sqrt{2 \pi} C \hbar^{4}}{3\left(k_{\mathrm{B}} T m^{*}\right)^{3 / 2} E^{2}},
$$


where $\hbar$ is the Planck constant, $k_{\mathrm{B}}$ is the Boltzmann constant, and $T$ is the absolute temperature. $C$ is the elastic constant which can be expressed as

$$
C=\frac{1}{V_{0}} \frac{\partial^{2} E}{\partial\left(\Delta l / l_{0}\right)^{2}},
$$

where $V_{0}$ and $l_{0}$ are the equilibrium volume and lattice constant of the unit cell. $\Delta l=l-l_{0}$ is the lattice strain and $E$ is the corresponding total energy of the system. When calculating the effective mass $\left(m^{*}\right)$ of electrons and holes, we have considered the band degeneracy. ${ }^{51}$ In formula (2), the deformation potential constant $(E)$ representing the conduction (valence) band edge shift per unit strain is given by

$$
E=\frac{\partial E_{\mathrm{edge}}}{\partial\left(\Delta l / l_{0}\right)} .
$$

To calculate the lattice thermal conductivity $\left(\kappa_{1}\right)$, we use the associated semi-empirical model ${ }^{52,53}$ from ground-state DFT calculations. In this approach, $\kappa_{1}$ is modeled as simple descriptors including acoustic $\left(\kappa_{1, \mathrm{ac}}\right)$ and optical phonon modes $\left(\kappa_{1, \mathrm{op}}\right) .{ }^{54}$ The predominant contribution stems from the acoustic phonon mode $\left(\kappa_{1, \mathrm{ac}}\right)$. Based on a simplified Debye-Callaway approach, the $\kappa_{1, \text { ac }}$ can be approximated to be

$$
\kappa_{\mathrm{l}, \mathrm{ac}}=\frac{\left(6 \pi^{2}\right)^{2 / 3}}{4 \pi^{2}} \frac{\bar{M} V_{\mathrm{s}}^{3}}{T V^{2 / 3} \gamma^{2} n^{1 / 3}},
$$

in which $\bar{M}$ and $V$ are the average mass and volume per atom respectively. $n$ is the number of total atoms in the primitive cell. The value of the Grüneisen constant $(\gamma)$ at different temperatures can be obtained from the crystal structure data by applying the quasi-harmonic Debye model. ${ }^{55,56} V_{\mathrm{s}}$ is the velocity of sound and is approximately equal to $\kappa_{1, \mathrm{op}}=\frac{3 k_{\mathrm{B}} V_{\mathrm{s}}}{2 V^{2 / 3}}\left(\frac{\pi}{6}\right)^{1 / 3}\left(1-\frac{1}{n^{2 / 3}}\right)$, where $B$ is the bulk modulus and $d$ is the density. The optical phonon mode is in the form of

$$
\kappa_{1, \mathrm{op}}=\frac{3 k_{\mathrm{B}} V_{\mathrm{s}}}{2 V^{2 / 3}}\left(\frac{\pi}{6}\right)^{1 / 3}\left(1-\frac{1}{n^{2 / 3}}\right) .
$$

All of these required parameters can be obtained from ground-state calculations.

\section{Results and discussion}

\subsection{Formation energy and structural stability}

We firstly assessed the structural stability of the $\mathrm{V}_{3} \mathrm{Al}_{1-x} \mathrm{Ga}_{x}(x=$ $0.25,0.5,0.75$ and 1) compounds based on the calculated formation energy. The formation energy of these doped compounds is in the form of

$$
E_{\mathrm{f}}=E_{\mathrm{V}_{3} \mathrm{Al}_{1-x} \mathrm{Ga}_{x}}+x E_{\mathrm{Al}}-\left(E_{\mathrm{V}_{3} \mathrm{Al}}+x E_{\mathrm{Ga}}\right)
$$

where $E_{\mathrm{V}_{3} \mathrm{Al}_{1-x} \mathrm{Ga}_{x}}$ and $E_{\mathrm{V}_{3} \mathrm{Al}}$ stand for the total energies of the doped compounds and the ideal $\mathrm{V}_{3} \mathrm{Al}$ per unit cell, $E_{\mathrm{Al}}$ and $E_{\mathrm{Ga}}$ are the chemical potentials of $\mathrm{Al}$ and $\mathrm{Ga}$ atoms respectively, and $x$ is the number of doped atoms. The calculated formation energies of all of the compounds are summarized in Table 1. Obviously, all the obtained values are negative, which indicates the thermodynamic stability of these doped compounds. The geometry structures of the $\mathrm{V}_{3} \mathrm{Al}_{1-x} \mathrm{Ga}_{x}(x=0.25,0.5,0.75$ and 1$)$ alloys in antiferromagnetic phase were then fully optimized based on energy minimization calculations. The configurations of the lowest energy were considered for further calculations. In Fig. 1, we present the total energy of $\mathrm{V}_{3} \mathrm{Al}_{1-x} \mathrm{Ga}_{x}(x=0.25,0.5$, 0.75 and 1) as a function of the strained lattice parameter with respect to the equilibrium parameter $\Delta l / l_{0}$ ranging from -0.05 to 0.05 . By fitting these calculated data to the third-order BirchMurnaghan equation of state (EOS), ${ }^{57,58}$ the optimal lattice parameters for all the compounds were obtained and are shown in Fig. 1 and collected in Table 1 . It is shown that the lattice constant decreases as the doping concentration increases from 0 to 1 in $\mathrm{V}_{3} \mathrm{Al}_{1-x} \mathrm{Ga}_{x}$, exhibiting good linear dependence and obeying Vegard's law. ${ }^{59}$ The obtained equilibrium lattice constants for $\mathrm{V}_{3} \mathrm{Al}$ and $\mathrm{V}_{3} \mathrm{Ga}$ are consistent with other theoretical and experimental results, ${ }^{\mathbf{2 8 , 2 9}}$ which indicates the reliability of the computational method used in this study. Unfortunately, we are not aware of available data to compare with our results for other doped compounds. In Table 1, we also present the anti-aligned atom-resolved magnetic moments (AMM). The $\mathrm{AMM}$ of $\mathrm{V}_{3}$ increases with an increase in the doping concentration whereas there is a decreasing trend for $\mathrm{V}_{2}$ due to the incorporation of $\mathrm{Ga}$ atoms in $\mathrm{V}_{3} \mathrm{Al}$.

\subsection{Mechanical stability and elastic properties}

The three independent elastic constants $\left(C_{11}, C_{12}\right.$, and $\left.C_{44}\right)$ for $\mathrm{V}_{3} \mathrm{Al}_{1-x} \mathrm{Ga}_{x}(x=0,0.25,0.5,0.75$ and 1$)$ are presented as

Table 1 The calculated formation energies $\left(E_{f}\right)$ (in eV), lattice constants (a) with available results (in $\AA$ ), and atom-resolved magnetic moments (AMM) (in $\mu_{\mathrm{B}}$ ) for $\mathrm{V}_{3} \mathrm{Al}_{1-x} \mathrm{Ga}_{x}(x=0,0.25,0.5,0.75$ and 1)

\begin{tabular}{llrrr}
\hline & $x$ & & & \\
\cline { 2 - 5 } & 0 & 0.25 & 0.5 & 0.75 \\
\hline$E_{\mathrm{f}}$ & & -0.049 & -0.090 & -0.124 \\
$a$ & $6.072\left(6.092^{a}, 6.06^{b}\right)$ & 6.064 & 6.056 & -0.151 \\
$\mathrm{AMM}\left(\mathrm{V}_{1}\right)$ & -1.527 & -1.509 & -1.482 & -1.460 \\
AMM $\left(\mathrm{V}_{2}\right)$ & 1.527 & 1.509 & 1.482 & 1.460 \\
$a$
\end{tabular}

${ }^{a}$ Experimental results in ref. 29. ${ }^{b}$ Theoretical results in ref. 28. 

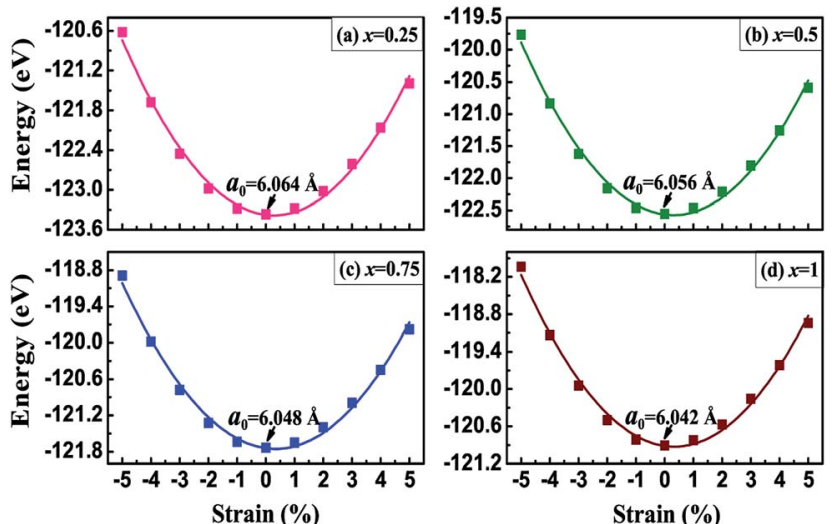

Fig. 1 The total energy as a function of the uniaxial strain $\Delta / / l_{0}$ with $\Delta l=l-l_{0}$ for $\mathrm{V}_{3} \mathrm{Al}_{1-x} \mathrm{Ga}_{x}$ alloys where (a) $x=0.25$, (b) $x=0.5$, (c) $x=$ 0.75 , and $(\mathrm{d}) x=1$.

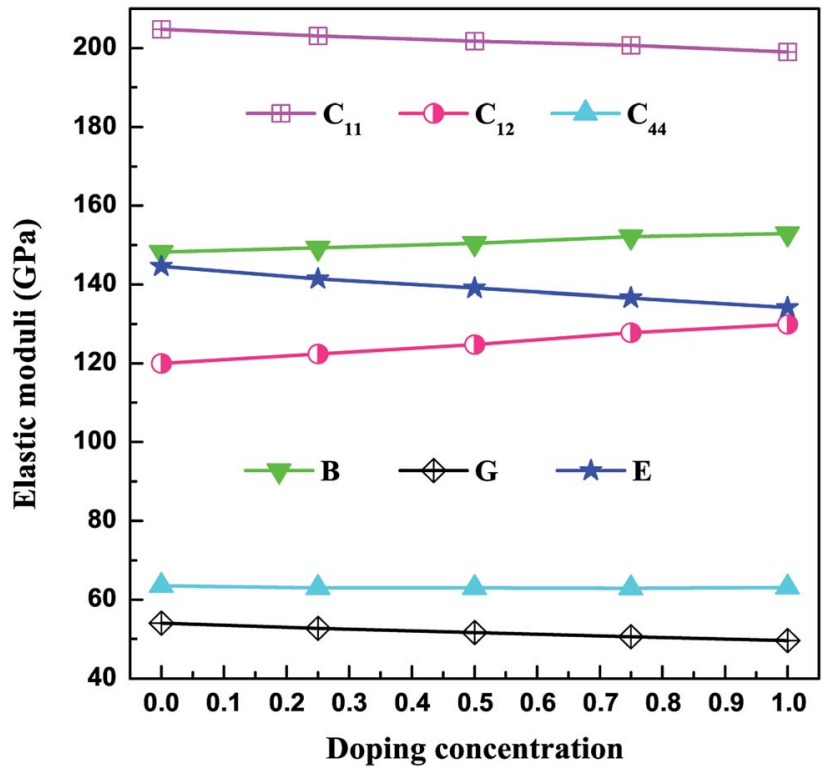

Fig. 2 Elastic constants and moduli as a function of the doping concentration.

a function of the doping concentration in Fig. 2 and Table 2. The considered compounds satisfy the mechanical stability criteria (1) and hence are mechanically stable. As is seen in Fig. 2, $C_{12}$ increases monotonically with the doping concentration, to which $C_{11}$ shows opposite variation. Meanwhile, $C_{44}$ is kept almost unvaried with the concentration of Ga. The obtained bulk modulus $(B)$, shear modulus $(G)$, and Young's modulus $(E)$ are also shown in Fig. 2. It is obvious that the value of $B$ shows an increasing trend as the doping concentration increases from $0\left(\mathrm{~V}_{3} \mathrm{Al}\right)$ to $1\left(\mathrm{~V}_{3} \mathrm{Ga}\right)$, indicating that it is more and more difficult to compress the listed compounds. The largest shear modulus $(G)$ for $\mathrm{V}_{3} \mathrm{Al}$ among all of the compounds means that $\mathrm{V}_{3} \mathrm{Al}$ can withstand the largest shear strain. As is well known, the Young's modulus $(E)$, defined as the ratio of stress and strain, is used to predict the stiffness of a material. The descending $E$ value of these compounds manifests that their stiffness becomes weaker with an increasing $x$. The ratio between the bulk modulus and the shear modulus $(B / G)$ is used to provide a measure of ductility or brittleness of a material. When $B / G$ is larger than 1.75 , the material is ductile, otherwise it is a brittle material. Our calculated $B / G$ values for the five alloys are all above 1.75 , indicating that they are prone to be ductile.

\subsection{Electronic properties}

Within the crystal structures at their equilibrium lattice constants, we further calculated the band structures and density of states (DOS) for the $\mathrm{V}_{3} \mathrm{Al}_{1-x} \mathrm{Ga}_{x}(x=0,0.25,0.5,0.75$ and 1) compounds. Fig. 3 shows the band structures and DOS for $x=0\left(\mathrm{~V}_{3} \mathrm{Al}\right)$ and $x=1\left(\mathrm{~V}_{3} \mathrm{Ga}\right)$. It can be seen from the left panel that the conduction band edge is very close to the valence band edge, forming narrow bandgaps of $0.08 \mathrm{eV}(x=0)$ and $0.06 \mathrm{eV}(x=1)$. The obtained bandgap values are both less than $0.1 \mathrm{eV}$, and therefore $\mathrm{V}_{3} \mathrm{Al}$ and $\mathrm{V}_{3} \mathrm{Ga}$ are antiferromagnetic gapless semiconductors. ${ }^{29}$ The experimental verification ${ }^{29}$ of such antiferromagnetic gapless semiconducting behavior of $\mathrm{V}_{3} \mathrm{Al}$ confirms the reliability of our calculations. In the right column of Fig. 3, the minimum of the conduction band is mainly derived from the $\mathrm{d}-\mathrm{d}$ hybridization between different $\mathrm{V}$ atoms and the maximum of the valence band is mostly contributed by the $3 \mathrm{~d}$ states of $\mathrm{V}$ atoms that possess positive magnetic moment. We then plotted the band structures and density of states for the doped systems $(x=0.25,0.5$ and 0.75$)$ in Fig. 4 . The change of the valence band maximum (VBM) and the conduction band minimum (CBM) with Ga substitution creates a small direct bandgap of $0.04 \mathrm{eV}$ for all of these doped systems, which is smaller than those of $\mathrm{V}_{3} \mathrm{Al}$ and $\mathrm{V}_{3} \mathrm{Ga}$. Such a small bandgap implies that all of the doped systems exist as gapless semiconductors. It can be seen from the DOS for these three doped systems that the states higher than the minimum of the conduction band are ascribed to the $3 \mathrm{~d}$ orbital of the $\mathrm{V}$ atoms

Table 2 Calculated elastic constants $\left(C_{i j}\right)$ and moduli $(B, G$, and $E)$ (all in $\left.G P a\right)$, and Grüneisen constants $(\gamma)$ for $V_{3} A_{1-x} G a_{x}(x=0,0.25,0.5,0.75$ and 1)

\begin{tabular}{llllllll}
\hline$x$ & $C_{11}$ & $C_{12}$ & $C_{44}$ & $B$ & $G$ & $B / G$ & $E$ \\
\hline 0 & 204.801 & 119.963 & 63.568 & 148.242 & 54.053 & 2.743 & 144.587 \\
0.25 & 203.056 & 122.354 & 62.968 & 149.255 & 52.679 & 2.833 & 141.400 \\
0.5 & 201.735 & 124.807 & 62.956 & 150.450 & 51.668 & 2.912 & 139.082 \\
0.75 & 200.722 & 127.786 & 62.930 & 152.098 & 20.559 & 3.008 & 136.548 \\
1 & 198.977 & 129.906 & 63.103 & 152.930 & 49.545 & 3.087 & 0.342 \\
& & & & & & 0.350 \\
& & & & & & & 134.149
\end{tabular}




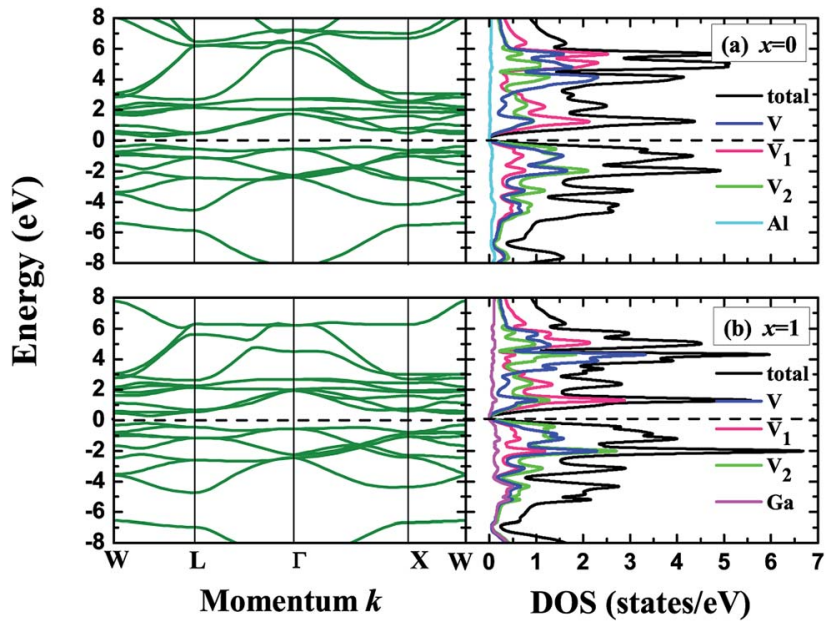

Fig. 3 Band structures (left), total and atom-projected density of states (right) for (a) $x=0\left(\mathrm{~V}_{3} \mathrm{Al}\right)$ and $(\mathrm{b}) x=1\left(\mathrm{~V}_{3} \mathrm{Ga}\right)$. The dashed lines denote the Fermi levels.

with a negative magnetic moment whereas the $3 \mathrm{~d}$ state hybridization between the other two $\mathrm{V}$ atoms forms most of that lower than the maximum of the valence bands. In addition, the energy band near the VBM and CBM of the doped systems that is flatter than that of $\mathrm{V}_{3} \mathrm{Al}$ suggests enhanced effective masses for all of the doped systems compared to $\mathrm{V}_{3} \mathrm{Al}$.

\subsection{Thermoelectric properties}

With the help of deformation potential theory, the obtained carrier relaxation times $(\tau)$ of $\mathrm{V}_{3} \mathrm{Al}_{1-x} \mathrm{Ga}_{x}(x=0.25,0.5,0.75$ and 1) for electrons and holes are summarized in Table 3 , together with the elastic constants $(C)$, deformation potential constants $(E)$, and effective masses $\left(m^{*}\right)$. The values of effective mass for electrons are smaller than those for holes in all of the systems, which is mainly derived from the sharper energy band around the CBM as shown in Fig. 3 and 4 . The varying effective mass as the doping concentration $(x)$ increases from 0 to 1 in $\mathrm{V}_{3} \mathrm{Al}_{1-x} \mathrm{Ga}_{x}$ is due to the significant influence on band structures made by substituting Ga for Al. Typically, $\tau=493$ fs for $n$-type $\mathrm{V}_{3} \mathrm{Al}$, and is almost 5 times as high as that for n-type $\mathrm{V}_{3} \mathrm{Al}_{0.25} \mathrm{Ga}_{0.75}$, and $\tau$ for p-type $\mathrm{V}_{3} \mathrm{Al}$ is $273 \mathrm{fs}$, which is about 2.7 times higher than that for p-type $\mathrm{V}_{3} \mathrm{Al}_{0.25} \mathrm{Ga}_{0.75}$. The low relaxation time in these $\mathrm{Ga}$ containing materials results from the spatially localized nature of the transition metal $3 \mathrm{~d}$ orbital. ${ }^{60}$ The experimental Néel temperature for $\mathrm{D}_{3} \mathrm{~V}_{3} \mathrm{Al}$ is $600 \mathrm{~K} .{ }^{29} \mathrm{~A}$ detailed study on the thermoelectric properties when the temperature ranges from 300 to $600 \mathrm{~K}$ was carried out in this section. However, theoretical computation showed that the Néel temperature for $\mathrm{DO}_{3} \mathrm{~V}_{3} \mathrm{Al}$ is as high as $988 \mathrm{~K}$ due to the large antiferromagnetic exchange interactions. ${ }^{31}$ As a result, the thermoelectric properties at $900 \mathrm{~K}$ are also given for comparison.

Fig. 5 shows the carrier concentration $(n)$ dependence of the Seebeck coefficient $(S)$ and the power factor (PF) at the temperatures of $300,450,600$, and $900 \mathrm{~K}$ for the $\mathrm{V}_{3} \mathrm{Al}_{1-x} \mathrm{Ga}_{x}(x=$ $0,0.25,0.5,0.75$ and 1) compounds. The absolute value of $S$ can reach a peak at a particular concentration for both p-type and n-
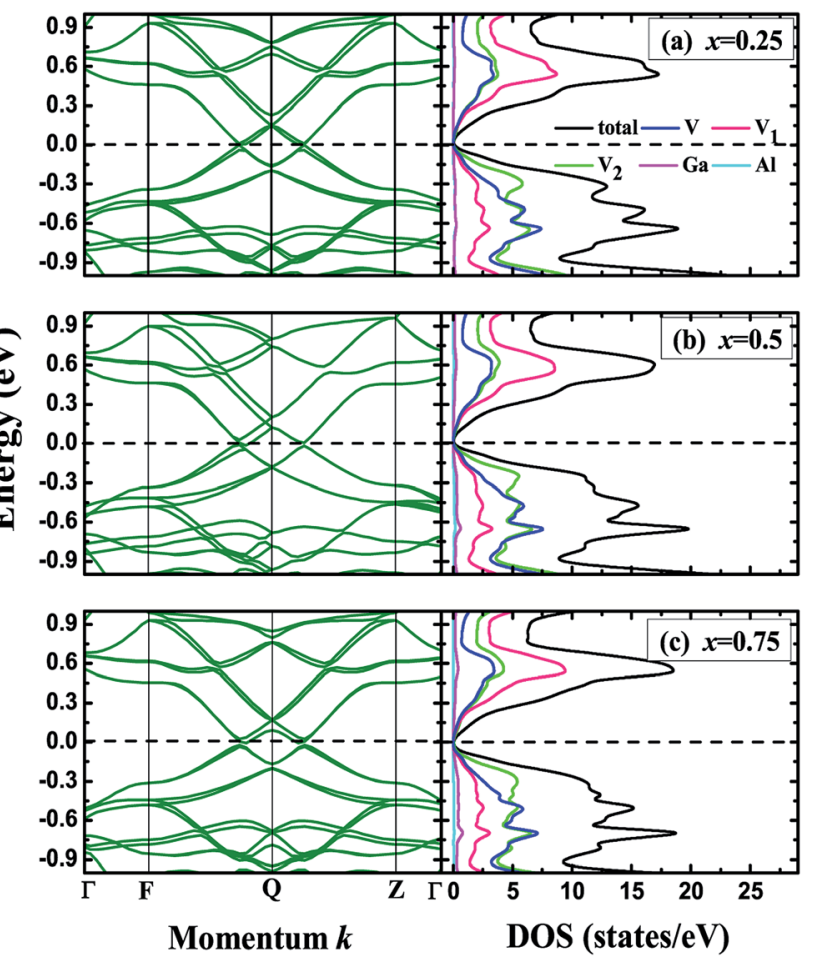

Fig. 4 Band structures (left), total and atom-projected density of states (right) for (a) $x=0.25$, (b) $x=0.5$ and (c) $x=0.75$. The dashed lines denote the Fermi levels.

type systems with an increasing $n$. The peak height becomes low and shifts slightly towards the higher carrier concentration with the enhancement of the temperature. It is obvious to find that the peak of the Seebeck coefficient at $300 \mathrm{~K}$ becomes higher with increasing $\mathrm{Ga}$ content in $\mathrm{V}_{3} \mathrm{Al}_{1-x} \mathrm{Ga}_{x}$. In addition, the highest absolute values of the Seebeck coefficient are obtained with $x=1$ for both $\mathrm{p}$-type and n-type systems. The peak values are $128.51(x=0)$ and $166.04 \mu \mathrm{V} \mathrm{K}^{-1}(x=1)$ for p-type systems, and $-112.57(x=0)$ and $-151.33 \mu \mathrm{V} \mathrm{K}^{-1}(x=1)$ for n-type systems. This indicates that the $\mathrm{DO}_{3} \quad \mathrm{~V}_{3} \mathrm{Ga}$ compound may have more favorable thermoelectric performance than $\mathrm{V}_{3} \mathrm{Al}$. It is clear that the calculated power factor first increases to a maximum and then is reduced with a further increase in the carrier concentration and it is expected that $\mathrm{PF}$ reaches the

Table 3 The calculated elastic constants $(C)$, deformation potential constants $(E)$, effective masses $\left(m^{*}\right)$, and obtained relaxation times $(\tau)$ at room temperature

\begin{tabular}{llllll}
\hline$x$ & Carrier type & $C$ & $E(\mathrm{eV})$ & $m^{*}\left(m_{\mathrm{e}}\right)$ & $\tau(\mathrm{fs})$ \\
\hline 0.25 & Electron & 1.459 & 27.595 & 0.071 & 355.602 \\
& Hole & 1.459 & 28.420 & 0.082 & 268.294 \\
0.5 & Electron & 1.471 & 27.241 & 0.129 & 150.160 \\
& Hole & 1.471 & 27.683 & 0.132 & 139.793 \\
0.75 & Electron & 1.483 & 26.575 & 0.166 & 108.191 \\
& Hole & 1.483 & 26.967 & 0.172 & 100.298 \\
1 & Electron & 1.498 & 26.560 & 0.153 & 123.751 \\
& Hole & 1.498 & 26.455 & 0.171 & 106.129
\end{tabular}



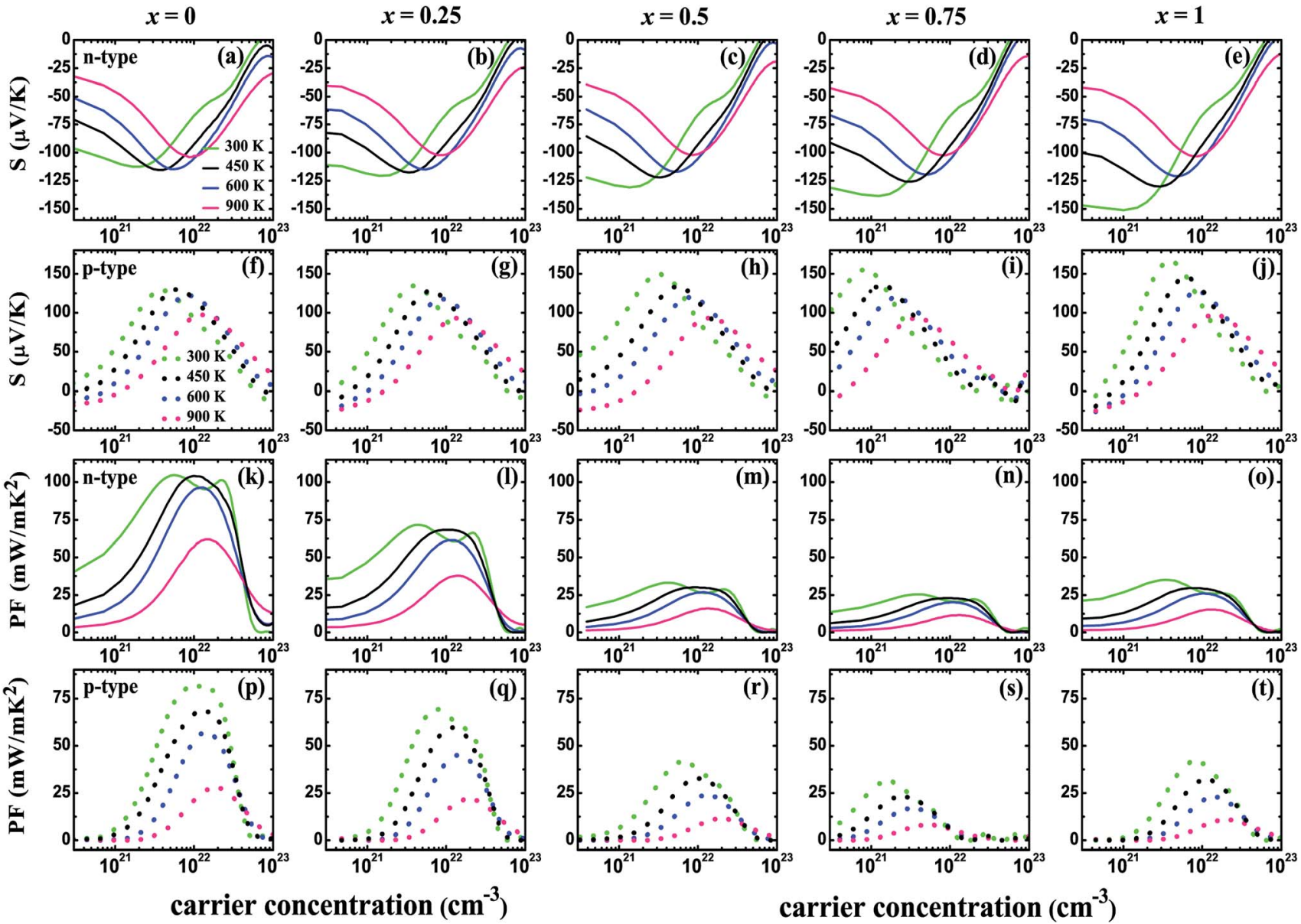

carrier concentration $\left(\mathrm{cm}^{-3}\right)$

\section{carrier concentration $\left(\mathrm{cm}^{-3}\right)$}

Fig. 5 The Seebeck coefficient $(S)((a-e)$ are for $n$-type systems and ( $f-j)$ are for $p$-type systems) and the power factor (PF) ((k-o) are for $n$-type systems and $(p-t)$ are for $p$-type systems) for $V_{3} \mathrm{Al}_{1-x} \mathrm{Ga}_{x}(x=0,0.25,0.5,0.75$ and 1$)$ as a function of the carrier concentration at temperatures of $300,450,600$, and $900 \mathrm{~K}$. Solid and dashed lines refer to results for $\mathrm{n}$-type and $\mathrm{p}$-type systems, respectively.

second maximum at high $n$ for all of the n-type systems at room temperature. The peak location gets smaller and shifts to the high carrier concentration range with increasing temperature. As the doping concentration increases from 0 to 0.75 , the increased effective mass leads to a higher Seebeck coefficient but contributes more to decreased carrier mobility. Therefore, the values of PF for the doped systems distinctly decrease with increasing effective mass. When $m^{*}$ is decreased in $\mathrm{V}_{3} \mathrm{Ga}(x=1)$, the optimal power factor increases. The thermal conductivities $(\kappa)$ and figures of merit $(z T)$ as a function of the carrier concentration $(n)$ at the temperatures of $300,450,600$, and 900 $\mathrm{K}$ for the $\mathrm{V}_{3} \mathrm{Al}_{1-x} \mathrm{Ga}_{x}(x=0,0.25,0.5,0.75$ and 1$)$ compounds are shown in Fig. 6 . The $\kappa$ values of the p-type systems are smaller than those of the n-type ones, which indicates that the thermoelectric performances of the n-type systems could be less favorable than those of the p-type ones. Fortunately, the increasing Ga content causes rapidly decreased total thermal conductivity over the measured temperatures due to the significant decrease in the lattice thermal conductivity. As a result, Ga doping of $\mathrm{V}_{3} \mathrm{Al}_{1-x} \mathrm{Ga}_{x}$ is an effective way to reduce the $\kappa$ of $\mathrm{V}_{3} \mathrm{Al}$. By inserting calculated thermoelectric transport parameters into $z T=S^{2} \sigma T / \kappa$, we can evaluate the thermoelectric performances of all of these compounds. The p-type systems exhibit larger $z T$ values than the n-type ones at 300,450 , and 600 $\mathrm{K}$, and it is completely different when the temperature is $900 \mathrm{~K}$, at which the largest $z T$ value is obtained for the n-type system. This indicates that good thermoelectric properties for all of the five compounds can be realized in p-type systems at 300, 450, and $600 \mathrm{~K}$. At the relatively high temperature of $900 \mathrm{~K}$, n-type compounds can be used as promising thermoelectric materials. When the temperature is varied from 300 to $900 \mathrm{~K}$, the maximal value of $z T$ firstly increases and then decreases. The optimal $z T$ values were $0.319(x=0), 0.298(x=0.25), 0.276(x=$ $0.5), 0.264(x=0.75)$, and $0.298(x=1)$ for p-type systems at 600 $\mathrm{K}$ and they were $0.297(x=0), 0.290(x=0.25), 0.267(x=0.5)$, $0.262(x=0.75)$, and $0.280(x=1)$ respectively for n-type systems. As discussed above, the changed band structure induced by Ga substitution leads to greatly decreased thermal conductivity although there is an undesirable decrease in power factor. So, an enhanced $z T$ could be anticipated in the $\mathrm{Ga}$ doping of $\mathrm{V}_{3} \mathrm{Al}_{1-x} \mathrm{Ga}_{x}$. It should be pointed out that $z T$ peaks at room temperature are significantly enhanced in some of the $\mathrm{Ga}$ doping systems. The highest obtained $z T$ values were $0.268(x=$ $0.25)$ and $0.275(x=1)$ for p-type systems, and $0.244(x=0.25)$ 

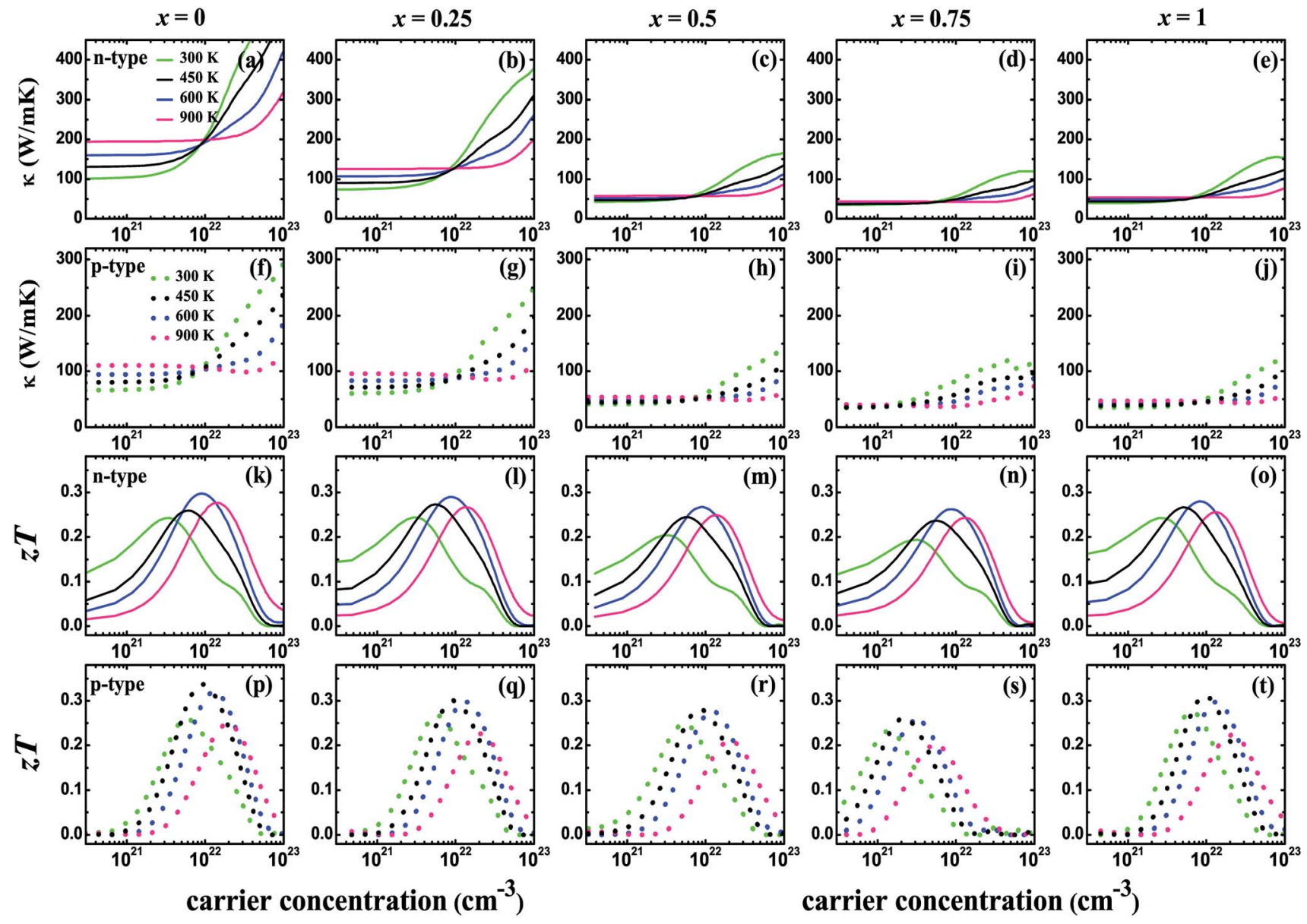

Fig. 6 The thermal conductivity $(\kappa)((a-e)$ are for $n$-type systems and $(f-j)$ are for $p$-type systems) and figure of merit $(z T)((k-0)$ are for $n$-type systems and $(\mathrm{p}-\mathrm{t})$ are for $\mathrm{p}$-type systems) for $\mathrm{V}_{3} \mathrm{Al}_{1-x} \mathrm{Ga}_{x}(x=0,0.25,0.5,0.75$ and 1$)$ as a function of the carrier concentration at temperatures of $300,450,600$, and $900 \mathrm{~K}$. Solid and dashed lines refer to results for $n$-type and $p$-type systems, respectively.

and $0.243(x=1)$ for n-type ones. Otherwise, the maximal $z T$ for pure $\mathrm{V}_{3} \mathrm{Al}$ is 0.259 (p-type) and 0.242 (n-type). Thus, in spite of having smaller power factors than pure $\mathrm{V}_{3} \mathrm{Al}, \mathrm{V}_{3} \mathrm{Al}_{0.75} \mathrm{Ga}_{0.25}$ and
$\mathrm{V}_{3} \mathrm{Ga}$ have higher $z T$ values because of the larger $S$ and lower $\kappa$. As a result, the thermoelectric performance of $\mathrm{V}_{3} \mathrm{Al}$ can be effectively improved by Ga doping. In Fig. 7, we present the
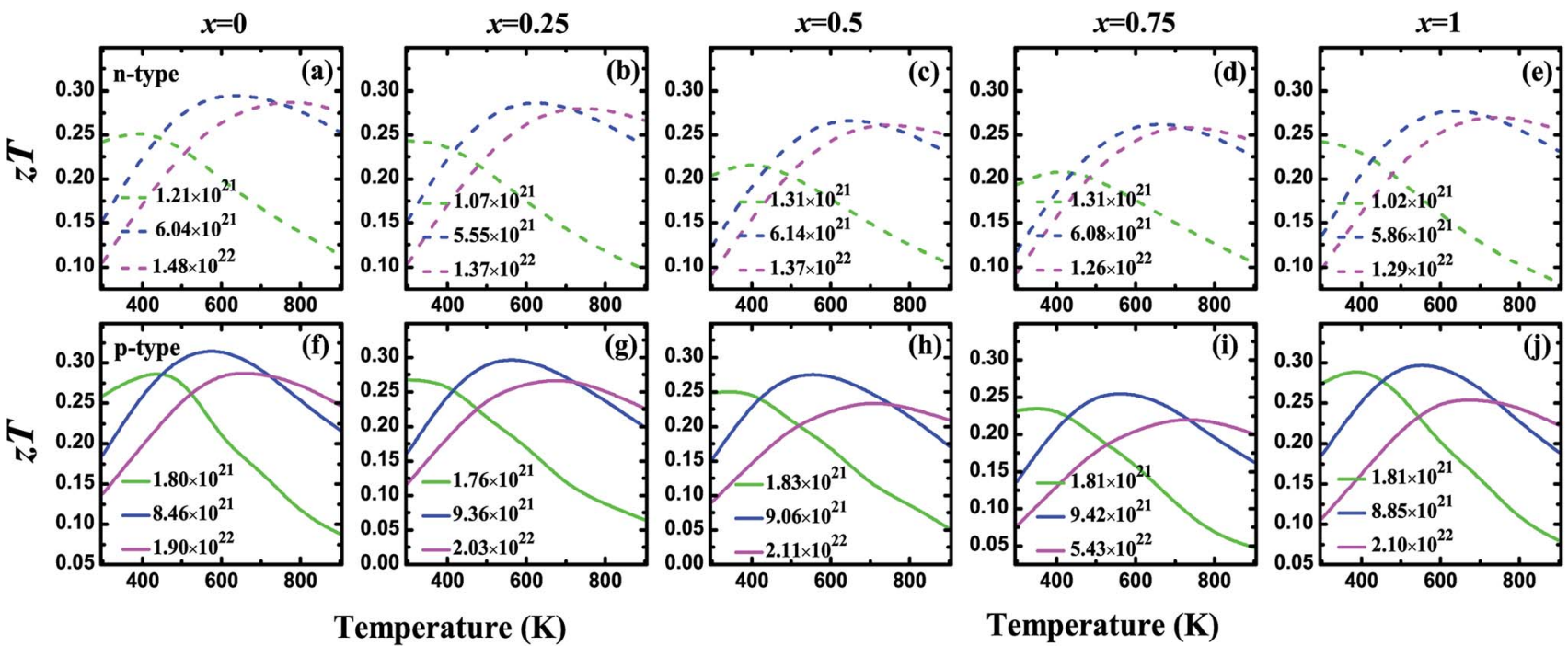

Fig. 7 The figure of merit $(z T)$ as a function of temperature for $n$-type $(a-e)$ and $p$-type $(f-j) V_{3} A_{1-x} G a_{x}(x=0,0.25,0.5,0.75$ and 1$)$ at four different optimal carrier concentrations. Solid and dashed lines refer to results for $n$-type and $p$-type systems, respectively. 
figure of merit $(z T)$ as a function of the temperature $(T)$ for both the n-type and p-type $\mathrm{V}_{3} \mathrm{Al}_{1-x} \mathrm{Ga}_{x}(x=0,0.25,0.5,0.75$ and 1$)$ compounds. Four optimal doping concentrations were chosen based on the maximum $z T$ at $300,450,600$, and $900 \mathrm{~K}$. From the variation of the figure of merit with temperature, the values of $T$ at which $z T$ achieves the maxima are higher with increasing carrier concentration and lies in the range of 500 to $700 \mathrm{~K}$. As such, p-type systems exhibit favorable TE performance even at low temperatures.

\section{Conclusions}

In this work, structural and mechanical stability, electronic structure and transport properties of $\mathrm{V}_{3} \mathrm{Al}_{1-x} \mathrm{Ga}_{x}(x=0.25,0.5$, 0.75 and 1) compounds have been investigated and compared with those of $\mathrm{V}_{3} \mathrm{Al}$ based on density functional theory combined with semi-classical Boltzmann theory. All of the studied systems were thermodynamically and mechanically stable. These compounds show narrow energy gaps of $0.04 \mathrm{eV}(x=0.25,0.5$ and 0.75$)$ and $0.06 \mathrm{eV}(x=1)$ and exist as antiferromagnetic gapless semiconductors. Ga substitution in pure $\mathrm{V}_{3} \mathrm{Al}$ makes the energy band near the CBM and VBM a little flatter, which results in an enhanced effective mass and a decreased power factor, but contributes more to decreased thermal conductivity. Thus, the maximal $z T$ is enhanced from 0.242 (n-type) and 0.259 (p-type) to $0.244(x=0.25)$ and $0.243(x=1)$ for $n$-type systems and $0.268(x=0.25)$ and $0.275(x=1)$ for $p$-type systems at room temperature.

\section{Conflicts of interest}

There are no conflicts to declare.

\section{Acknowledgements}

This work was supported by the National Natural Science Foundation of China under Grant No. 11875226 and 11874306, the Natural Science Foundation of Chongqing under Grant No. CSTC-2017jcyjBX0035, and the Postgraduates' Research and Innovation Project of Chongqing No. CYB17077.

\section{Notes and references}

1 G. D. Mahan and J. O. Sofo, Proc. Natl. Acad. Sci. U. S. A., 1996, 93, 7436-7439.

2 F. J. DiSalvo, Science, 1999, 285, 703-706.

3 S. Chen and Z. F. Ren, Mater. Today, 2013, 16, 387-395.

4 M. Schwall and B. Balke, Appl. Phys. Lett., 2011, 98, 042106.

5 C. Uher, J. Yang, S. Hu, D. T. Morelli and G. P. Meisner, Phys. Rev. B, 2009, 59, 8615-8621.

6 W. Xie, Q. Jin and X. Tang, J. Appl. Phys., 2008, 103, 043711.

7 M. Zhou, L. D. Chen, C. D. Feng, D. L. Wang and J. F. Li, J. Appl. Phys., 2007, 101, 113714.

8 C. Uher, J. Yang, S. Hu, D. T. Morelli and G. P. Meisner, Phys. Rev. B, 1999, 59, 8615-8621.
9 G. Joshi, R. He, M. Engber, G. Samsonidze, T. Pantha, E. Dahal, K. Dahal, J. Yang, Y. C. Lan, B. Kozinsky and Z. F. Ren, Energy Environ. Sci., 2014, 7, 4070-4076.

10 S. Bhattacharya, T. M. Tritt, Y. Xia, V. Ponnambalam, S. J. Poon and N. Thadhani, Appl. Phys. Lett., 2002, 81, 43-45. 11 C. Yu, T. J. Zhu, R. Z. Shi, Y. Zhang, X. B. Zhao and J. He, Acta Mater., 2009, 57, 2757-2764.

12 L. Chen, S. Gao, X. Zeng, A. M. Dehkordi, T. M. Tritt and S. J. Poon, Appl. Phys. Lett., 2015, 107, 041902.

13 S. Sakurada and N. Shutoh, Appl. Phys. Lett., 2005, 86, 082105.

14 M. Schwall and B. Balke, Phys. Chem. Chem. Phys., 2013, 15, 1868.

15 C. G. Fu, S. Q. Bai, Y. T. Liu, Y. S. Tang, L. D. Chen, X. B. Zhao and T. J. Zhu, Nat. Commun., 2015, 6, 8144.

16 C. Fu, T. Zhu, Y. Liu, H. Xiea and X. Zhao, Energy Environ. Sci., 2015, 8, 216.

17 Y. Nishino, S. Deguchi and U. Mizutani, Phys. Rev. B, 2006, 74, 115115.

18 M. Mikami, Y. Kinemuchi, K. Ozaki, Y. Terazawa and T. Takeuchi, J. Appl. Phys., 2012, 111, 093710.

19 K. Renard, A. Mori, Y. Yamada, S. Tanaka, H. Miyazaki and Y. Nishino, J. Appl. Phys., 2014, 115, 033707.

20 T. Zou, T. Jia, W. Xie, Y. Zhang, M. Widenmeyer, X. Xia and A. Weidenkaff, Phys. Chem. Chem. Phys., 2017, 19, 1827318278.

21 I. Pallecchi, M. Pani, F. Ricci, S. Lemal, D. I. Bilc, P. Ghosez, C. Bernini, N. Ardoino, G. Lamura and D. Marre, Phys. Rev. Mater., 2018, 2, 075403.

22 M. Vasundhara, V. Srinivas and V. V. Rao, Phys. Rev. B, 2008, 77, 224415.

23 H. Miyazaki, S. Tanaka, N. Ide, K. Soda and Y. Nishino, Mater. Res. Express, 2014, 1, 015901.

24 H. Miyazaki, M. Inukai and Y. Nishino, J. Appl. Phys., 2016, 120, 125106.

25 X. M. Li, L. Tao, Z. F. Chen, H. Fang, X. S. Li, X. R. Wang, J. B. Xu and H. W. Zhu, Appl. Phys. Rev., 2017, 4, 021306.

26 P. Qiu, J. Yang, X. Huang, X. Chen and L. Chen, Appl. Phys. Lett., 2010, 96, 152105.

27 M. L. C. Buffon, G. Laurita, L. Lamontagne, E. E. Levin, S. Mooraj, D. L. Lloyd, N. White, T. M. Pollock and R. Seshadri, J. Phys.: Condens. Matter, 2017, 29, 405702.

28 G. Y. Gao and K. L. Yao, Appl. Phys. Lett., 2013, 103, 232409. 29 M. E. Jamer, B. A. Assaf, G. E. Sterbinsky, D. Arena, L. H. Lewis, A. A. Saul, G. Radtke and D. Heiman, Phys. Rev. B, 2015, 91, 094409.

30 X. R. Chen, Y. H. Huang and H. Chen, RSC Adv., 2017, 7, 44647-44654.

31 I. Galanakis, S. Tirpanci, K. Ozdogan and E. Sasioglu, Phys. Rev. B, 2016, 94, 064401.

32 Y. L. Han, Z. B. Chen, M. Q. Kuang, Z. H. Liu, X. J. Wang and X. T. Wang, Results Phys., 2019, 12, 435-446.

33 P. E. Blochl, Phys. Rev. B, 1994, 50, 17953-17979.

34 G. Kresse and J. Hafner, Phys. Rev. B, 1993, 47, 558-561.

35 G. Kresse and J. Hafner, Phys. Rev. B, 1994, 49, 14251-14269.

36 G. Kresse and J. Furthmuller, Comput. Mater. Sci., 1996, 6, 15-50. 
37 J. P. Perdew, K. Burke and M. Ernzerhof, Phys. Rev. Lett., 1996, 77, 3865-3868.

38 X. R. Chen, M. M. Zhong, Y. Feng, Y. Zhou, H. K. Yuan and H. Chen, Phys. Status Solidi B, 2015, 252, 2830-2839.

39 Y. Li, H. K. Yuan, J. H. Xia, G. L. Zhang, M. M. Zhong, A. L. Kuang, G. Z. Wang, X. R. Zheng and H. Chen, Eur. Phys. J.: Appl. Phys., 2015, 70, 31001.

40 E. Haque and M. A. Hossain, Results Phys., 2018, 10, 458-465.

41 R. Hill, Proc. Phys. Soc., London, Sect. A, 1952, 65, 349-355.

42 G. V. Sinko and N. A. Smirnov, J. Phys.: Condens. Matter, 2002, 14, 6989-7005.

43 S. Yip, J. Li, M. J. Tang and J. G. Wang, J. Mater. Sci. Eng. A, 2001, 317, 236-240.

44 A. N. Gandi and U. Schwingenschlogl, Chem. Mater., 2014, 26, 6628-6637.

45 V. K. Gudelli, V. Kanchana, G. Vaitheeswaran, D. J. Singh, A. Svane, N. E. Christensen and S. D. Mahanti, Phys. Rev. $B, 2015,92,045206$.

46 S. Q. Hao, F. Y. Shi, V. P. Dravid, M. G. Kanatzidis and C. Wolverton, Chem. Mater., 2016, 28, 3218-3226.

47 K. P. Ong, D. J. Singh and P. Wu, Phys. Rev. B, 2011, 83, 115110.

48 A. J. Hong, L. Li, R. He, J. J. Gong, Z. B. Yan, K. F. Wang, J. M. Liu and Z. F. Ren, Sci. Rep., 2016, 6, 22778.
49 Y. Q. Cai, G. Zhang and Y. W. Zhang, J. Am. Chem. Soc., 2014, 136, 6269-6275.

50 J. Bardeen and W. Shockley, Phys. Rev., 1950, 80, 72-80.

51 M. A. Green, J. Appl. Phys., 1990, 67, 2944-2954.

52 J. Yan, P. Gorai, B. Ortiz, S. Miller, S. A. Barnett, T. Mason, V. Stevanovic and E. S. Toberer, Energy Environ. Sci., 2015, 8, 983-994.

53 J. Callaway, Phys. Rev., 1959, 113, 1046-1051.

54 E. S. Toberer, A. Zevalkink and G. J. Snyder, J. Mater. Chem., 2011, 21, 15843-15852.

55 E. Francisco, J. M. Recio, M. A. Blanco, A. M. Pendas and A. Costales, J. Phys. Chem. A, 1998, 102, 1595-1601.

56 A. Zevalkink, D. M. Smiadak, J. L. Blackburn, A. J. Ferguson, M. L. Chabinyc, O. Delaire, J. Wang, K. Kovnir, J. Martin, L. T. Schelhas, T. D. Sparks, S. D. Kang, M. T. Dylla, G. J. Snyder, B. R. Ortiz and E. S. Toberer, Appl. Phys. Rev., 2018, 5, 021303.

57 F. D. Murnaghan, Proc. Natl. Acad. Sci. U. S. A., 1944, 30, 244247.

58 F. Birch, Phys. Rev., 1947, 71, 809-824.

59 L. Vegard, Proc. Natl. Acad. Sci. U. S. A., 1921, 5, 17-26.

60 J. Yang, P. Qiu, R. Liu, L. Xi, S. Zheng, W. Zhang, L. Chen, D. J. Singh and J. H. Yang, Phys. Rev. B, 2011, 84, 235205. 